maintaining the tegretol have shown conclusively that it is the PA and fish oil that are responsible for the recovery.

PA is a food additive aka E-304 (EU) or ascorbyl palmitate (USA) and as a powerful, lipid soluble anti-oxidant is widely used in the food industry as a preservative and freshener in meats and salads. It is cheap ( $€ 45 / \mathrm{kg}$ ) and safe (LD50 3,000 $\mathrm{mg} / \mathrm{kg}$ ). It is freely available in bulk or personal quantities online.

The result shown in this case is similar to that we have found in some cases of rheumatoid arthritis with rapid reductions in swelling and pain in finger joints and long-term reduction of back pain.

This result must be of interest to researchers and to dentists and their patients with intractable trigeminal neuralgia. We look forward to an explanation in due course. I will be happy to correspond with any interested parties on andrew@brainhelp.info.

A. Carmichael

By email

DOI: 10.1038/sj.bdj.2012.324

\section{NEW REVIEW NEEDED}

Sir, the number of papers proposing a link between periodontal disease and systemic disease is ever increasing. The latest area under investigation is breast cancer. A recent study by Soder et al. ${ }^{1}$ looked at whether patients with periodontal disease have an increased breast cancer incidence if they had lost molar teeth. The inference was that the missing molars were purely an indication of the previous severity of the periodontal disease. What if the extraction sites themselves were the source of the increased disease incidence? Lechner and Mayer $^{2}$ investigated areas of poorly healed extraction sites and found them to contain pro-inflammatory mediators such as IL1-ra and RANTES. These lesions were histologically an avascular necrosis of the medullary bone and have been reported in the dental literature as far back as 1915 by G. V. Black. If this is the case then it may be that some of the previous papers on periodontal disease and systemic disease need to be reviewed in a new light.

\section{J. Ahearne, Poole}

1. Söder B, Yakob M, Meurman J H, Andersson L $C$, Klinge B, Söder $P$ Ö. Periodontal disease may associate with breast cancer. Breast Cancer Res Treat 2011; 127: 497-502.

2. Lechner J, Mayer W. Immune messengers in Neuralgia Inducing Cavitational Osteonecrosis (NICO) in jaw bone and systemic interference. Europ J Integrative Med 2010; 2: 71-77.

DOI: 10.1038/sj.bdj.2012.325

\section{AN ALTERNATIVE REACTION}

Sir, I am writing to bring attention to the case of a 47-year-old male smoker initially diagnosed with aggressive periodontitis. The patient presented with what was deemed to be approximately $70 \%$ generalised horizontal bone loss affecting his remaining teeth (Fig. 1). Upon looking at the radiograph closely, particularly at the lower jaw, there does not appear to be loss of bone height per se in the mandible, just a loss of bone surrounding the teeth, making the teeth appear extruded - see height of bone above inferior dental canal and anterior mandible. In light of this and other similar observations, could tooth extrusion be an alternative reaction of the periodontium to the effects of dental neglect, smoking and other patient factors?

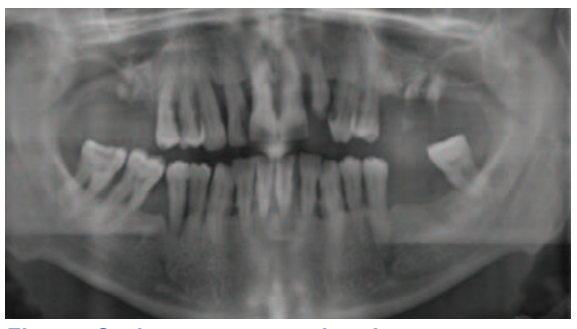

Fig. 1 Orthopantogram showing 70\% generalised horizontal bone loss

V. Weblin, J. Yates, Manchester DOI: 10.1038/sj.bdj.2012.326

\section{STRUGGLE FOR EXPERIENCE}

Sir, I am currently studying for my A-levels and hoping to pursue a career in dentistry. I have been actively seeking work experience for a while now and have contacted many dentists in my area with no success. The most frequent responses are: ' $m y$ insurance will not allow it' and 'there are issues with confidentiality that prevent me from allowing it'. Although I can completely sympathise with the practitioners, how are the potential dentists of the future to gain much needed experience of their preferred profession?

I hope this letter may encourage your readers to give students the opportunity they need, even if it only involves sitting on reception and a chat at lunch.

T. Williams, Chester tomwilliams_93@hotmail.com DOI: 10.1038/sj.bdj.2012.327

\section{CONTROLLABLE FLUORIDE}

Sir, I am writing in response to the letter from A. McKay published on your website on 9 March (Organic toothpaste; $B D J$ 2012; 212: 206) in which the correspondent criticises our fluoride-free children's toothpaste, claiming that this product is 'potentially harmful', 'unnecessary', and that dental care professionals should 'discourage its use'.

We are well aware of the undoubted benefits of fluoride in the prevention of dental caries, and the fact that many children are deficient in this essential mineral. However, we are also aware of the potential adverse effects of excessive fluoride intake, in particular the risk of dental fluorosis and the psychological impact that this can have on children.

Taking into account the widely differing fluoride intakes due to regional variations in water fluoridation and dietary intakes, we feel that fluoride supplementation should be assessed on an individual basis rather than given in a rather arbitrary way through toothpaste. In particular, we are concerned that the dosage levels achieved through toothpaste are prone to very wide fluctuations. The amount of toothpaste used, the frequency of use, whether the toothpaste is spat out or swallowed, and whether the mouth is rinsed after brushing will all affect the potential dose of fluoride received from this route.

We believe that a safer and more accurately controllable means of administration is through the use of fluoride tablets or drops which can be given to children in need of supplementation with this mineral. Assuming that is done, there is no reason why children should need to use a fluoridated toothpaste with the attendant risk of excess fluoride intake that these bring, and instead can benefit from using our totally natural and certified organic toothpastes.

I. Taylor

Information \& Research Manager, Green People Organic Lifestyle DOI: 10.1038/sj.bdj.2012.328 